Published in final edited form as:

Comput Methods Biomech Biomed Engin. 2013 October ; 16(10): 1112-1126. doi:

10.1080/10255842.2013.809711.

\title{
Evaluation of a Post-Processing Approach for Multiscale Analysis of Biphasic Mechanics of Chondrocytes
}

\author{
Scott C. Sibole ${ }^{1,2}$, Steve Maas ${ }^{3,4}$, Jason P. Halloran ${ }^{1,2}$, Jeffrey A. Weiss ${ }^{3,4,5}$, and Ahmet \\ Erdemir $^{1,2}$ \\ ${ }^{1}$ Computational Biomodeling (CoBi) Core, Lerner Research Institute, Cleveland Clinic, Cleveland, \\ Ohio, USA \\ ${ }^{2}$ Department of Biomedical Engineering, Lerner Research Institute, Cleveland Clinic, Cleveland, \\ Ohio, USA \\ ${ }^{3}$ Department of Bioengineering, University of Utah, Salt Lake City, Utah, USA \\ ${ }^{4}$ Scientific Computing and Imaging Institute, University of Utah, Salt Lake City, Utah, USA \\ ${ }^{5}$ Department of Orthopaedics, University of Utah, Salt Lake City, Utah, USA
}

\begin{abstract}
Understanding the mechanical behavior of chondrocytes as a result of cartilage tissue mechanics has significant implications for both evaluation of mechanobiological function and to elaborate on damage mechanisms. A common procedure for prediction of chondrocyte mechanics (and of cell mechanics in general) relies on a computational post-processing approach where tissue level deformations drive cell level models. Potential loss of information in this numerical coupling approach may cause erroneous cellular scale results, particularly during multiphysics analysis of cartilage. The goal of this study was to evaluate the capacity of $1^{\text {st }}$ and $2^{\text {nd }}$ order data passing to predict chondrocyte mechanics by analyzing cartilage deformations obtained for varying complexity of loading scenarios. A tissue scale model with a sub-region incorporating representation of chondron size and distribution served as control. The postprocessing approach first required solution of a homogeneous tissue level model, results of which were used to drive a separate cell level model (same characteristics as the subregion of control model). The $1^{\text {st }}$ data passing appeared to be adequate for simplified loading of the cartilage and for a subset of cell deformation metrics, e.g., change in aspect ratio. The $2^{\text {nd }}$ order data passing scheme was more accurate, particularly when asymmetric permeability of the tissue boundaries were considered. Yet, the method exhibited limitations for predictions of instantaneous metrics related to the fluid phase, e.g., mass exchange rate. Nonetheless, employing higher-order data exchange schemes may be necessary to understand the biphasic mechanics of cells under lifelike tissue loading states for the whole time history of the simulation.
\end{abstract}

Corresponding Author: Ahmet Erdemir, PhD Department of Biomedical Engineering (ND20) Lerner Research Institute Cleveland Clinic phone: +1 (216) 4459523 fax: +1 (216) 4449198 erdemira@ @cf.org.

7. Appendix

Appendices A. Taylor Series Quantities, B. Element Volume Calculation, C. Mass Transport Integration, and D. Median and Range for Chondrocyte Mechanics Metrics can be accessed through the online edition as supplementary material. 


\section{Keywords}

multiscale; computational modeling; finite element; cartilage; chondrocyte; poroelastic; biphasic; tissue mechanics; cell mechanics; homogenization

\section{Introduction}

Chondrocytes, the sole cell type found in cartilage, provide the basis for cartilage growth, maintenance, and overall health. Chondrocyte response and activity is regulated by a complex interaction between many factors including, genetic, environmental, and biomechanical factors (Grodzinsky et al., 2000). Biomechanical factors include but are not limited to tension, compression, shear, osmolarity, fluid pressure, and associated electrokinetic effects (Alexopoulos et al., 2005; Soltz and Ateshian, 1998; Urban, 2000). To understand the in vivo mechanical environment of chondrocytes, one must consider the multiscale load transfer from the body, to the tissue, and then to the cell.

Development of tools for quantification of chondrocyte level mechanics, and potentially biological response, is an area of ongoing research. Proposed methods include two predominant areas, computational or experimental (Halloran et al., 2012). Experimental studies have provided much of the fundamental information on cartilage biomechanics, but cannot resolve the complete internal mechanical state of this tissue and its cells. Likewise, there are considerable barriers to multiscale investigation of cartilage mechanics through experimentation due to the large disparity in measurement resolution needed at difference spatial scales and limitations to quantify different cell mechanical metrics. Consequentially, computational investigations of cartilage and chondrocytes have become the tool of choice for interpreting the biomechanical and biophysical basis of experimental results, and as an independent investigative approach when experimental investigation is difficult or not practical (Goldsmith et al., 1996; Guilak and Mow, 2000; Mow et al., 1993; Soulhat et al., 1999).

Multiscale computational modeling and simulation approaches for quantification of chondrocyte mechanics commonly rely on a post-processing analysis. In such a procedure, the analysis begins with the solution of a boundary value problem at the tissue-scale. A cell scale model, ideally representative of chondrocyte shape, size and distribution, is then solved with tissue-scale mechanics dictating the loading and boundary conditions (Guilak and Mow, 2000). The means to inform a cell-scale model's boundary conditions from tissuescale deformations usually require assumptions for mechanical coupling and may have significant influence on simulation results, particularly when the complicated multiphysics are considered. When only a small set of points within the cartilage are of interest, an obvious choice is to overlay a cell scale model within a macroscopic model, and calculate appropriate boundary conditions by interpolation from appropriate field variables in the tissue-scale model. This approach has been useful to provide insight into mechanics of chondrocytes through simulations conducted for several points within a cartilage model (Guilak and Mow, 2000; Moo et al., 2012) and has also been utilized for tissue constructs (Yan et al., 2010). However, the implementation constraints associated with this approach 
may hinder streamlined analysis. Interpolations involving a large set of macro-scale and micro-scale nodes may increase computational cost and book-keeping efforts. Further, implementation issues may arise when the overlay of the cell-scale model results in some of the surface nodes being located outside the geometric boundaries of the tissue-scale model, e.g., for the superficial zone of the cartilage.

An alternative approach for post-processing utilizes tissue-scale mechanical information at a given point to approximate loading and boundary conditions of a cell-scale model, in the biphasic case, the deformation gradient and fluid pressure. Adapted from computational homogenization techniques (Kouznetsova et al., 2004), this approach streamlines large scale analyses, e.g., many points in a tissue-scale model, as illustrated by the quantification of elastic deformations of chondrocytes for large sections of tibial and femoral cartilage (Sibole and Erdemir, 2012). The aforementioned interpolation technique is replaced by a Taylorseries approximation, which only relies on the information from the finite element containing the point of interest in the macro-scale model. A $1^{\text {st }}$ order approximation is common; it has been used to estimate chondrocyte mechanics from cartilage strains (Sibole and Erdemir, 2012) and to explore cell mechanics in other tissues such as the meniscus (Upton et al., 2006) and the intervertebral disk (Cao et al., 2011). However, when the relative sizes of the cell-scale model and tissue characteristic length are comparable, higher order approximations may be necessary to capture the nonlinearities over the cell-scale model volume (Kouznetsova et al., 2004).

The method, by which the down-scale communication of mechanical information is accomplished, and the resulting translation into micro-scale boundary conditions can strongly affect performance and fidelity, depending on the nature of the higher scale boundary value problem. This has been generally characterized for elastic materials (Kouznetsova et al., 2004). However, to the best of the authors' knowledge, no assessment has been made for biphasic materials, in general, or for the specific case of tissue-cellular scale modeling in cartilage. In addition, there is a need to quantify the resulting uncertainty in different cell metrics, which may have varying importance for the interpretation of potential cell damage and mechanical stimuli. Therefore, the overall goal of this study is to understand how the assumptions of multiscale data transfer during post-processing of tissue mechanics (specifically cartilage) influence the solid and fluid mechanics of cells (specifically chondrocytes). Provided a control case, the specific aims are to quantify the uncertainty in predicted biphasic mechanics of cells i) for $1^{\text {st }}$ and $2^{\text {nd }}$ order information passing from the tissue scale, and ii) under four different loading cases representing increased complexity of tissue level elastic deformations and fluid boundary conditions. For tissue mechanics in general, and for cartilage mechanics specifically, the study establishes appropriate procedures for estimation of cell level deformations when tissue level mechanics are known, i.e., quantified experimentally or predicted through computational modeling.

\section{Methods}

\subsection{Models}

All numerical models employed a biphasic constitutive model for the material components: extracellular matrix (ECM), pericellular matrix (PCM), and chondrocyte (Table 1). The 
solid phase of these materials was defined as isotropic neo-Hookean. The fluid phase was modeled with strain-independent, isotropic hydraulic permeability directly relating the fluid pressure gradient to fluid flux via Darcy's Law (Maas et al., 2012).

2.1.1 Embedded Model-To serve as a control model for the study, a cellular-scale mesh was embedded at the center of a larger cube of ECM ( $1 \mathrm{~mm}$ edge length) containing a total of 152868 linear hexahedral elements (Figure 1A). Essentially, this was a tissue-scale model of the transitional zone of cartilage with anatomically-based cellular-scale representation for a small region at its center (Hunziker et al., 2002). Only the transitional zone was considered in the current study, as it allowed for the gross assumption of material isotropy for the extracellular matrix and the cartilage. The cellular-scale mesh was a cube of ECM (100 $\mu \mathrm{m}$ edge length) that contained a geometric representation of 11 spherical chondrocytes with 10 $\mu m$ diameters, each surrounded by a $2.5 \mu m$ thick PCM. Nodes on the surface of the cellularscale mesh were directly merged with the tissue-scale mesh, circumventing the need to define numerical interaction constraint(s), e.g., tied surfaces or contact.

\subsubsection{Autonomous Models}

2.1.2.1 Tissue-scale Model: A homogeneous tissue-scale model consisted of a $1 \mathrm{~mm}$ edge length cube of ECM discretized with 42875 linear hexahedral elements (Figure 1B). Lacking the representation of the heterogeneous cellular-scale geometry, this model made the assumption that the contribution of the chondrocytes and PCM was negligible to the tissuescale response. It should be noted that in the $100 \mu \mathrm{m}$ cube with cellular representation, $94.2 \%$ of the volume was ECM. The element at the center of the mesh had an edge length of $0.2 \mathrm{~mm}$. This resulted in a scale ratio between the finite element of interest in the tissue-scale model and the size of the cellular-scale model of $2^{n}: 1$ where $n$ was the spatial dimension considered, e.g., $8: 1$ for three-dimensional. Likewise, the total tissue-cell scale separation was $10^{n}: 1$. The mesh for this model was refined until the average mechanical response of the centroid element very closely matched the average mechanical response of the microscale region in the control model.

2.1.2.2 Cellular-scale Model: The mesh embedded at the center of the control model also served as a separate cellular-scale model to be used in the post-processing coupling approach (Figure 1C). This geometry was discretized with 97725 linear hexahedral elements. The mesh density was based on the results of a convergence study for a single chondrocyte model (Sibole et al., 2012).

\subsection{Coupling}

For simulations of chondrocyte mechanics, which utilize autonomous models at the tissuescale and cellular-scale, a post-processing based numerical coupling approach was adopted. If the deformation gradient is assumed to be homogeneous across the cellular-scale volume, the material to spatial frame mapping of surface node positions at a given time, $t$, is

$$
\boldsymbol{x}(\boldsymbol{X}, t)=\boldsymbol{F}(\boldsymbol{X}, t) \cdot \boldsymbol{X}, \quad(1)
$$


where $\boldsymbol{x}(t)$ are the spatial and $\boldsymbol{X}$ are the material frame position vectors and the deformation gradient is defined as

$$
\boldsymbol{F}(\boldsymbol{X}, t)=\frac{\partial \boldsymbol{x}(\boldsymbol{X}, t)}{\partial \boldsymbol{X}} .
$$

This homogeneous mapping is equivalent to a $1^{\text {st }}$ order Taylor series such that

$$
\boldsymbol{x}(\boldsymbol{X}, t)=f(\boldsymbol{X}, t)=f(\mathbf{0}, \mathbf{t})+\frac{\partial f(\boldsymbol{X}, t)}{\partial \boldsymbol{X}} \cdot \boldsymbol{X}+\boldsymbol{O}\left(\boldsymbol{X}^{\mathbf{2}}\right),
$$

where the $1^{\text {st }}$ term, $f(0, t)$, corresponds to rigid body motion and can be omitted. The error associated with this approximation can be reduced by considering the 2nd order Taylor series approximation

$$
\boldsymbol{x}(\boldsymbol{X}, t)=f(\boldsymbol{X}, t)=f(\mathbf{0}, \mathbf{t})+\frac{\partial f(\boldsymbol{X}, t)}{\partial \boldsymbol{X}} \cdot \boldsymbol{X}+\frac{1}{2} \boldsymbol{X}^{\boldsymbol{T}} \cdot \frac{\partial f(\boldsymbol{X}, t)}{\partial \boldsymbol{X}^{2}} \cdot \boldsymbol{X}+\boldsymbol{O}\left(\boldsymbol{X}^{\mathbf{3}}\right) .
$$

This allows for the representation of non-homogeneous deformations as

$$
\boldsymbol{x}(\boldsymbol{X}, t)=F(\boldsymbol{X}, t) \cdot \boldsymbol{X}+\frac{1}{2} \boldsymbol{X}^{\boldsymbol{T}} \cdot \boldsymbol{G}(\boldsymbol{X}, t) \cdot \boldsymbol{X},
$$

where the $3^{\text {rd }}$ order tensor,

$$
\boldsymbol{G}(\boldsymbol{X}, t)=\frac{\partial^{2} \boldsymbol{x}(\boldsymbol{X}, t)}{\partial \boldsymbol{X}^{2}},
$$

is the deformation Laplacian.

For a poroelastic model, one must also communicate the fluid pressure state from the tissuescale down to the cellular-scale. Again, this can be approximated with a $1^{\text {st }}$ order

$$
p(\boldsymbol{X}, t)=p(\mathbf{0}, \mathbf{t})+\frac{\partial p(\boldsymbol{X}, t)}{\partial \boldsymbol{X}} \cdot \boldsymbol{X} \quad(7)
$$

or $2^{\text {nd }}$ order

$$
p(\boldsymbol{X}, t)=p(\mathbf{0}, \mathbf{t})+\frac{\partial p(\boldsymbol{X}, t)}{\partial \boldsymbol{X}} \cdot \boldsymbol{X}+\frac{1}{2} \boldsymbol{X}^{\boldsymbol{T}} \cdot \frac{\partial^{2} p(\boldsymbol{X}, t)}{\partial \boldsymbol{X}^{2}} \cdot \boldsymbol{X}
$$

Taylor series. In Equations 7 and 8, $p(\boldsymbol{0}, t)$, accounts for the current fluid pressure acting uniformly across the finite volume. This is analogous to rigid body motion, but in contrast, cannot be omitted due to its contribution to the total stress state of the volume. From a model solution at the macro-scale, in this case the tissue-scale, one can calculate $\boldsymbol{F}(\boldsymbol{X}, t)$, $\boldsymbol{G}(\boldsymbol{X}, t), \mathrm{p}(\mathbf{0}, \mathrm{t}), \frac{\partial p(\boldsymbol{X}, t)}{\partial \boldsymbol{X}}$, and $\frac{\partial^{2} p(\boldsymbol{X}, t)}{\partial \boldsymbol{X}^{2}}$ at any location within each finite element using the isoparametric shape functions and their spatial derivatives (see Appendix A). These quantities are exact for the infinitesimal volume local to this position in the tissue-scale model. Equations 3 and 7 can then be used to approximate the deformed surface nodal 
positions and fluid pressures on the finite volume of a micro-scale model with $1^{\text {st }}$ order Taylor series accuracy. Likewise, Equations 4 and 8 will supply $2^{\text {nd }}$ order accuracy.

The material to spatial frame position mappings were applied to the cellular-scale model as surface node displacement boundary conditions such that

$$
\boldsymbol{u}(\boldsymbol{X}, t)=\boldsymbol{x}(\boldsymbol{X}, t)-\boldsymbol{X}, \quad(9)
$$

where $\boldsymbol{x}(\boldsymbol{X}, t)$ was determined with either $1^{\text {st }}$ or $2^{\text {nd }}$ order accuracy from Equations 3 or 4 , respectively, and $\boldsymbol{X}$ were the coordinates of the cellular-scale model surface nodes in the material frame. $\boldsymbol{p}(\boldsymbol{X}, t)$ from Equations 7 or 8 serves as the fluid pressure boundary condition without any additional calculation.

\subsection{Cell Level Mechanical Variables}

For both the embedded model and post-processing based simulations using autonomous models, mechanics of individual chondrocytes were characterized at each simulation time point with the following metrics: change in chondrocyte aspect ratio, volumetric strain, the volume-averaged effective stress, effective strain, and maximum shear strain, and the net cellular fluid mass exchange rate. The motivation behind using these metrics was to supply a considerably diverse array of metrics so as to create an extensive picture of the chondrocyte mechanics. Some metrics were indicative of the solid phase response of the material, others the fluid phase, and yet others, a combination of both solid and fluid phases. The volumes of the finite elements comprising each chondrocyte were calculated from nodal positions at each time point using the isoparametric formulation and Gaussian quadrature (Appendix B).

2.3.1 Change in Chondrocyte Aspect Ratio-The ellipsoid of best fit was calculated for each chondrocyte over time by assembling the mass moment of inertia tensor for chondrocyte element sets (Peeters et al., 2004). Assuming unit density, the mass moment of inertia tensor, $\boldsymbol{I}(t)$, was calculated with

$$
\boldsymbol{I}(t)=\sum_{e} V_{e}(t)\left[\begin{array}{ccc}
y_{e}(t)^{2}+z_{e}(t)^{2} & -x_{e}(t) y_{e}(t) & -x_{e}(t) z_{e}(t) \\
-y_{e}(t) x_{e}(t) & x_{e}(t)^{2}+z_{e}(t)^{2} & -y_{e}(t) z_{e}(t) \\
-z_{e}(t) x_{e}(t) & -z_{e}(t) y_{e}(t) & x_{e}(t)^{2}+y_{e}(t)^{2}
\end{array}\right],
$$

where $V_{e}(t)$ was the volume and $x_{e}(t), y_{e}(t), z_{e}(t)$ were the components of the centroid position vector of element $e$ at time $t$.

The eigenvalues, $\lambda_{i}(t)$ in descending order, of this tensor are the principal moments of inertia. These can be used to determine the length of the axes of the ellipsoid of best fit, $L_{i}(t)$ in ascending order by

$$
L_{i}(t)=\frac{1}{\sqrt{\lambda_{i}(t)}}
$$

These axes were used to determine major-minor radii $\left(L_{3}\right.$ and $L_{1}$, respectively), providing the bounds of overall chondrocyte shape, i.e., the limit case. From these, changes in chondrocyte aspect ratios at each time point were calculated as 


$$
\kappa_{31}(t)=\frac{L_{3}(t)}{L_{1}(t)}-\frac{L_{3}(0)}{L_{1}(0)}
$$

2.3.2 Volumetric Strain-Volumetric strain for each chondrocyte was determined as

$$
\epsilon_{v}=\frac{\sum_{e} V_{e}(t)}{\sum_{e} V_{e}(0)}-1
$$

2.3.3 Average Effective Stress and Strain-The effective stress and strain were calculated from the Cauchy stress and Green-Lagrange strain tensors at the 8 Gaussian integration point for each element in chondrocyte set using

$\sigma_{\text {effective }}(t), \epsilon_{\text {effective }}(t)=\sqrt{\frac{1}{2}\left[\left(\lambda_{1}(t)-\lambda_{2}(t)\right)^{2}+\left(\lambda_{2}(t)-\lambda_{3}(t)\right)^{2}+\left(\lambda_{3}(t)-\lambda_{1}(t)\right)^{2}\right]}$,

where $\lambda_{1}(t), \lambda_{2}(t)$, and $\lambda_{3}(t)$ are the eigenvalues of the stress or strain tensors corresponding to the first, second, and third principal stresses or strains at time $t$. These 8 values were then averaged to get a mean element value.

The effective stresses and strains are positive semi-definite functions; therefore, averaging over chondrocyte volume using

$$
\bar{\psi}(t)=\frac{\sum_{e} V_{e}(t) \psi_{e}(t)}{\sum_{e} V_{e}(t)}
$$

was valid. Here, $\psi_{e}(t)$ is any positive semi-definite function calculated for element $e$, at time $t$.

2.3.4 Average Maximum Shear Strain-The maximum shear strain was calculated from the Green-Lagrange strain tensor at chondrocyte element integration points as

$$
\tau_{\max }(t)=\max \left\{\left|\lambda_{2}(t)-\lambda_{3}(t)\right| \quad\left|\lambda_{3}(t)-\lambda_{1}(t)\right| \quad\left|\lambda_{1}(t)-\lambda_{2}(t)\right|\right\}
$$

and averaged across each element. These were also volume-averaged with Equation 15 to obtain the average value for each chondrocyte.

2.3.5 Mass Transport-The net mass exchange rate at solution time points, entering or exiting the chondrocyte volume, was determined from the fluid fluxes reported at the finite element nodes. Through application of Gauss' Divergence Theorem to a chondrocyte volume for fluid flux, the equality

$$
\iiint_{V} \nabla \cdot \boldsymbol{q}(t) d V=\oint \oint_{S} \boldsymbol{q}(t) \cdot \boldsymbol{n}(t) d S
$$


where $\boldsymbol{q}(t)$ and $\boldsymbol{n}(t)$ respectively represent the fluid flux and surface normals of a chondrocyte at time $t$, can be obtained.

By defining surface sets for each chondrocyte, it was possible to extract fluid fluxes at nodes on the surface of chondrocytes. Nodal fluid fluxes and local surface normals were interpolated over the surface facets to obtain values at the integration points via the isoparametric formulation for quadrilateral facets (Appendix C). The mass exchange rate at time $t$, was obtained through calculation of the surface integral corresponding to the righthand side of Equation 17 using Gaussian quadrature such that

$$
\dot{m}=\rho \sum_{e} \sum_{i}^{4} \boldsymbol{q}_{\boldsymbol{i}}(t) \cdot \boldsymbol{n}_{\boldsymbol{i}}(t) \omega_{i}(t),
$$

where $\boldsymbol{q}_{i}(t), \boldsymbol{n}_{i}(t)$, and $\omega_{i}(t)$ were the fluid flux, surface normal, and Gaussian weight at integration point $i$ on facet $e$, respectively, and $\rho$ was the density of water $\left(1 \times 10^{9} \mathrm{pg} / \mathrm{mm}^{3}\right)$.

\subsection{Simulation Cases}

To evaluate the performance of the post-processing approach for varying complexity of loading and boundary conditions, four different simulation cases were considered (Figure 2). All simulations were conducted using FEBio version 1.5.0 (Maas et al., 2012).

2.4.1 Case A-The first case consisted of confined compression stress relaxation in the $z$ direction - $0.1 \mathrm{~mm}$ compression linearly ramped over 0.1 seconds and held constant for 99.9 seconds with free-draining of fluid on top and bottom faces. This scenario resulted in symmetric fluid flux and displacement about the model $z$-midline. Simulation results were sampled at $0.0,0.01,0.05,0.1,0.15,0.2,0.5,1,2,3,4,5,6,7,8,9,10,15,20,25,30,35$, $40,45,50,60,70,80,90$, and 100 seconds.

2.4.2 Case B-This case incorporated the same conditions as Case A, except fluid could only drain from the top face. This scenario resulted in asymmetric fluid flux and displacement about the model $z$-midline.

2.4.3 Case C-Again, this case used the same conditions as Case A, except displacement of top face was non-uniform and defined by a nonlinear mapping

$$
z=Z-\frac{1}{2} \sqrt{(X)}-\frac{1}{2} \sqrt{(Y)}
$$

where $\mathrm{X}, \mathrm{Y}$, and $\mathrm{Z}$ were the components of the undeformed nodal position vectors. This scenario considered a non-uniform surface displacement with nearly symmetric fluid behavior.

2.4.4 Case D-This case consisted of the same conditions as Case C, except fluid could only drain from the top face. This scenario considered a non-uniform surface displacement with asymmetric fluid behavior. 


\subsection{Evaluation of Coupling Assumptions}

The performance of the $1^{\text {st }}$ and $2^{\text {nd }}$ order post-processing approaches were evaluated for all chondrocyte mechanical metrics using several measures comparing predictions of autonomous models against those of the embedded control model. Some of these calculations utilized the whole time history of cell response, while others considered only specific time points.

2.5.1 Root-Mean-Square Differences-The root-mean-square differences between $1^{\text {st }}$ order data passing and control and between $2^{\text {nd }}$ order data passing and control were calculated throughout the whole time history with information from all cells combined:

$$
R M S_{\text {control } \rightarrow \text { coupled }}=\sqrt{\left(\frac{\sum_{1}^{C} \sum_{0}^{N}\left(\lambda_{\text {coupled }}-\lambda_{\text {control }}\right)^{2}}{C N}\right)},
$$

where $N$ is the number of time-points, $C$ is the number of chondrocytes and $\lambda$ refers to a given chondrocyte mechanical metric. Root-mean-square differences were also normalized by the range of the metric obtained from the control simulation, $\mid \max \left(\lambda_{\text {control }}\right)-$ min $\left(\lambda_{\text {control }}\right.$.

2.5.2 Bland-Altman Limits of Agreement-The limits of agreement according to Bland and Altman (Altman and Bland, 1983) were determined for $1^{\text {st }}$ and $2^{\text {nd }}$ order versus the control for the whole simulation duration, for 11 cells on a cell-by-cell basis at each time instant. The number of values lying outside the $95 \%$ limits of agreement were recorded for each comparison.

2.5.3 Distribution of Chondrocyte Mechanics Metrics-Metrics to describe chondrocyte mechanics were also extracted from two time points of loading, referred to as "instantaneous" and "steady state", and for the "peak" of cellular mechanical response regardless of its occurrence through the course of loading (Figure 3). The instantaneous response corresponded to the time instant of tissue displacement reaching its maximal value $(0.1 \mathrm{~s})$, to explore the fast-loading mechanics of chondrocytes. The steady state response corresponded to the final simulation time $(100 \mathrm{~s})$, to quantify the equilibrium state of the chondrocytes. The peak values of chondrocyte mechanics can have significance for evaluation of potential damage mechanisms or to quantify maximum mechanical stimuli transferred to the cell. The data from 11 cells did not exhibit a normal distribution, based on qualitative interpretation of their probability density function plots. Therefore, median values and ranges for instantaneous, steady-state, and peak response were reported.

2.5.4 Correlation-For each simulation case, Spearman rank correlation coefficients (Spearman, 2010) were calculated for each cellular metric at instantaneous, steady-state and peak values between $1^{\text {st }}$ order data passing and control and $2^{\text {nd }}$ order data passing and control using R (Ihaka and Gentleman, 1996). Correlation analysis relied on the correspondence of mechanical metrics for each individual cell, in this case 11 chondrocytes. 
To compare the correlation coefficients calculated for first and second-order methods, Fisher's Transform was used to determine a $z$-score and the corresponding $p$-value (Fisher, 1921).

\section{Results}

As intended, the simulations driven by each of the four boundary condition cases resulted in markedly different mechanics at the level of the chondrocytes (Figures $4 \& 5$ ). The influence of boundary conditions was apparent for metrics descriptive of both solid and fluid components of poroelastic simulations. Qualitative comparison of the time evolution of chondrocyte metrics in the control, $1^{\text {st }}$, and $2^{\text {nd }}$ order simulations provided substantial insight into the performance of the two coupling methods (Figures $4 \& 5$ ). For boundary condition Cases B and D (asymmetric permeability conditions), qualitative differences were more apparent between chondrocyte metrics reported by the 1 st and $2^{\text {nd }}$ order simulations with the $2^{\text {nd }}$ order approach displaying better agreement with the control. Large differences were not qualitatively observable between the two approaches for Case A and Case C (symmetric permeability conditions).

Quantitatively, the $2^{\text {nd }}$ order data passing approach followed predictions of the control more closely, as illustrated by the RMS differences (Table 2). The overall improvement in performance using $2^{\text {nd }}$ order data passing was also apparent as the number of predictions outside the limits of agreement were generally lower (Figure 6), particularly with increased complexity of loading and boundary conditions. An exception to the better agreement of the $2^{\text {nd }}$ order was the mass exchange rate, which showed an increased RMS difference for the $2^{\text {nd }}$ order data passing for Cases $\mathrm{C} \& \mathrm{D}$. Potential artifacts of utilizing $2^{\text {nd }}$ order data passing were noted, particularly when specific time points (instantaneous, steady-state, and peak) for chondrocyte metrics were evaluated. For example, the correlation coefficients calculated for mass exchange rate and volumetric strain at the instantaneous time point were significantly worse for the $2^{\text {nd }}$ order approximation (Table 3 ). The instantaneous mass exchange rate was also the peak value for Cases $C \& D$. The median and range of each variable at the instantaneous, peak, and steady-state time points were reported in Appendix D.

\section{Discussion}

In general, the results of this study indicated that the $2^{\text {nd }}$ order Taylor series approach to data passing between scales exhibited better agreement with the control simulations than the $1^{\text {st }}$ order approach, particularly when the whole duration of a loading case is considered. The need for a $2^{\text {nd }}$ order approach, for prediction of cell deformation metrics, was more apparent when loading and boundary conditions for the macro-scale tissue model were more complicated, e.g., for case D (asymmetric fluid pressure and non-uniform displacement) (Figure 2). Asymmetric fluid permeability conditions potentially induced a more nonuniform fluid pressure gradient over the cellular region, as indicated by the non-zero fluid pressure Laplacian. The resulting fluid flux interacted with the solid phase through drag forces and impacted the internal displacement field of the cellular region, which already exhibited complexities due to non-uniform boundary conditions at the tissue scale. As a result, chondrocyte mechanics were strongly influenced. The ability of the $2^{\text {nd }}$ order 
approximation to capture this nonuniformity resulted in its superior agreement with the control model when deformation metrics were considered.

For a given study, quantification of chondrocyte mechanics at particular time points may be more important than the entire time history of chondrocyte mechanics. For instance, peak deformation would be applicable to a cellular damage study, instantaneous response would be relevant for highly dynamic activities such as impacts, and steady-state response would be relevant for cases of prolonged static loading. The relevance of these time points were variable-specific. For example, at the first instance of full ramp displacement, chondrocytes essentially behaved as nearly incompressible materials and thus volumetric strain and other deformation metrics were very close to zero (Appendix D). Similarly, at steady-state, by definition the fluid flux would be zero and thus mass exchange rate should also be zero (Appendix D). These limiting behaviors are expected for biphasic materials (Ateshian et al., 2007). Comparison of metrics in such cases may reflect errors largely attributable to machine precision and round-off errors. Accordingly, correlation between $1^{\text {st }}$ or $2^{\text {nd }}$ order data passing and control at these time points were not sought after (Table 3).

For specific time-points, it was interesting to note the deficiency of $2^{\text {nd }}$ order data passing to predict metrics of chondrocyte mechanics, particularly those associated with the fluid phase. Superiority of the $2^{\text {nd }}$ order approach for prediction of peak effective stress (Table 3, Cases B \& D) and maximum shear strain (Table 3, Case B) was not reflected for instantaneous mass exchange rate and volumetric strain (Table 3, Cases C \& D). At the instantaneous time-point, the volumetric strain values were lower than other time points, e.g. median of -0.004 (Table D.1 in Appendix D), which may have contributed to this artifact. Peak mass exchange rate values also showed discrepancies, simply because peak and instantaneous time points for this variable were the same. It may have been possible that the $2^{\text {nd }}$ order data passing scheme resulted in an over fitting of fluid pressure boundary conditions acquired from the macro-scale simulations, and therefore in the application of artificially increased and non-uniform fluid pressure boundary conditions. As a result, chondrocyte mass exchange rates during the ramp loading phase were over predicted (Figure 7). Further, the fluid flux field within the cell region, despite visually closer to the control case, spatially varied in a different manner (Figure 8). This situation may have influenced one-to-one correspondence of cellular mass exchange rates and related volumetric strains. This issue may have also resulted in higher RMS differences in predicted mass exchange rate for the $2^{\text {nd }}$ order data passing (Table 2). Yet, in view of limits of agreement over the whole simulation time history, the $2^{\text {nd }}$ order data passing was more adequate (Figure 6). Therefore, the instantaneous time point should be considered as a special case. One may utilize different data passing orders for fluid and solid phases, i.e., $1^{\text {st }}$ for the former and $2^{\text {nd }}$ order for the latter. This may resolve the issues related to the prediction of instantaneous metrics. Additionally, if scale separation between macro and micro scales is suspected as a source of error, the order of data passing can be increased further.

The post-processing approach, $1^{\text {st }}$ or $2^{\text {nd }}$ order, should be robust against nonuniform loading and boundary conditions, which are likely to occur during the analysis of in vivo cartilage mechanics. The non-uniform displacement field in the cellular-scale region induced solely by a nonlinear displacement mapping of the top face in Case $\mathrm{C}$ did not require $2^{\text {nd }}$ order 
approximation to produce the resulting chondrocyte mechanics in the control simulation as evidenced by the minimal differences between $1^{\text {st }}$ and $2^{\text {nd }}$ order. Likewise, the simplest boundary condition, Case A, did not require $2^{\text {nd }}$ order accuracy. Nonetheless, in situ cartilage loading may not resemble such conditions (Mononen et al., 2012). The $2^{\text {nd }}$ order data passing approach, which can more robustly represent the loading and boundary conditions at the cell-scale, may be safer to use for investigation of the functional mechanics of chondrocytes.

The realism of the models for the representation of actual cartilage and chondrocyte anatomy, microstructure, and constitutive properties were limited in this study. While physiological accuracy was sacrificed, this simplification allowed for the reduction of computational cost during the evaluation of the effect of non-uniformities in loading and boundary conditions on post-processing based quantification of chondrocyte metrics. The latter was indeed one goal of the study, also supporting development and cross evaluation of $1^{\text {st }}$ and $2^{\text {nd }}$ order data passing in post-processing based multiscale analysis. One should consider this work as a methodology demonstration, upon which more complicated cases can be evaluated with different model parameters and constitutive representations. The freely available dissemination package accompanying this manuscript will aid in such an endeavor (see Dissemination).

With regard to cartilage anatomy, although the models were morphometrically representative of transitional zone cartilage based on histological statistics (Hunziker et al., 2002), the cellular and pericellular geometries were idealized as spheres with no variation in cellular diameters or pericellular thicknesses. The cellular membrane was also not considered, and the interface between the chondrocyte and PCM was assumed to be perfectly adhesive, as opposed to focally attached at integrin binding sites (Schwartz, 2010). The laminar structure of cartilage (superficial, transitional, and deep zones) including its transition to calcified bone was not considered. The constitutive models assigned to the ECM, PCM, and chondrocytes did not incorporate strain dependent anisotropy, due to changing alignment of the initially randomly-oriented collagen fibril network of transitional zone cartilage (Ateshian et al., 2009), but rather assumed isotropic behavior at all strains. Likewise, the fibrous structure of the PCM (Julkunen et al., 2009) was also not represented, nor was any anisotropy resulting from the cellular cytoskeleton (Chen et al., 2012). The fluid permeability was assumed to be strain-independent and isotropic, yet permeability has been shown to decrease with increasing strain due to reduction in porosity and increase in tortuosity (Holmes and Mow, 1990). In reality, the relative permeability between the ECM and chondrocytes may be different than the one used in this study. i.e., with cells having a higher permeability than ECM. This ratio was known to influence transient cell deformations particularly when the aggregate modulus of the cell and extracellular matrix were similar (Guilak and Mow, 2000), which was not the case in the models of this study. Nevertheless, constitutive models incorporating some or all of these properties could be used in a future study without additional modification to the coupling approach. So-called triphasic modeling of the movement of charged particles within the cartilage and the resultant osmotic effects (Lai et al., 1991; Wang et al., 2002) was not considered. Extending 
to this degree of realism would require modifications to the presented technique to account for communication of the ion content between scales.

Increased complexity for the representation of cartilage and chondrocyte physics and physiology will likely increase non-uniformity of field variables at a cellular region interest. Therefore, it will challenge the performance of the data passing approach to move from the spatial scale of cartilage to that of the chondrocytes. As it was observed for the loading and boundary conditions (Figure 6 ), the performance gap between $1^{\text {st }}$ and $2^{\text {nd }}$ order data passing will likely widen with more realistic representation of material properties and fiber architecture. It is also possible that $2^{\text {nd }}$ order performance may degrade, indicating a need for even higher order approximations or even preclude the use of a post-processing approach. The post-processing based multiscale analysis framework will allow the exploration of full thickness cartilage, with macroscopic delineation of zones, including their depth-dependent permeability and fiber organization. In following, different cell scale models, representative of individual zones can be solved and chondrocyte metrics can be calculated. Such an analysis is important to establish realism of multiscale cartilage mechanics. Yet, it was beyond the scope of this first assessment of the data passing approach.

Additional limitations are associated with model generation and analysis of the results. To allow for comparison of results between simulations, results were sampled at specific time points. Therefore, aliasing errors were likely introduced. To reduce such errors, resolution of time sampling was higher early in the simulation, when dynamic effects would be dominant. Relevant to time sampling, one should also note that RMS error gave a time-aggregate measure of differences between control, $1^{\text {st }}$, and $2^{\text {nd }}$ order techniques, which may be influenced by this time sampling and may not be a good measure of agreement at specific times. The analysis to establish limits of agreement between data passing schemes and control may also be affected in a similar manner. For this reason, chondrocyte mechanics were also evaluated at multiple time points, e.g., instantaneous, and steady-state, with each offering a different scope of applicability. In terms of spatial discretization, difficulty in hexahedral mesh generation due to the geometric complexity of the inclusion of randomly placed chondrons imposed limitation on obtaining an optimal discretization of the cellularscale boundary value problem. While a mesh convergence study was conducted, there may be additional discretization artifacts that may impact simulated chondrocyte mechanics. Nonetheless, the effect of such errors can be assumed to be similar between control simulations and $1^{\text {st }}$ and $2^{\text {nd }}$ order data passing approaches.

This study used a large set of metrics to characterize chondrocyte mechanics. These metrics characterized overall cell deformation (aspect ratio, volumetric strain, effective strain, maximum shear strain) but also included overall cell stress state (effective stress) and fluid flow (mass exchange rate). The deformation metrics may provide insight into chondrocyte damage mechanisms, and offer complimentary information to existing experimental capabilities. Utilizing multiple metrics provides the possibility to assess chondrocyte damage from combined deformation modes, and are also useful to characterize the various types of mechanical stimuli acting on the cells. Metrics describing fluid flow may be indicators of potential chemical signaling between neighboring cells. Through the 
exploration of a large set of cell variables, differences were observed between $1^{\text {st }}$ and $2^{\text {nd }}$ order approximations that may have gone unnoticed if only a subset of metrics were calculated. For example, if only changes in chondrocyte aspect ratios were considered, differences between the $1^{\text {st }}$ and $2^{\text {nd }}$ order data passing schemes would have appeared small. However, effective strain and effective stress show marked differences between the methods (Figures 4 and 5). This serves as somewhat of a cautionary tale for data analysis of chondrocyte mechanics in either experimental or computational realms, i.e., characterization of mechanics requires informed use of a given metric, or studies should strive to use a diverse array of variables when possible. Conversely, if change in aspect ratio is the only variable of interest, a $1^{\text {st }}$ order approximation scheme may be sufficient (Figures $4 \& 5$ ).

The study findings regarding the accuracy of $1^{\text {st }}$ and $2^{\text {nd }}$ order post-processing schemes are applicable to other multiscale coupling approaches as well. For example, bi-directional multiscale modeling techniques obtain a solution at higher and lower scales synchronously with simulation progression dependent on information passing between scales at each time step. Computational homogenization is a wide-spread example of a bi-directional technique (Geers et al., 2010), during which the constitutive behavior at each integration point in the higher scale problem is determined by the average stress tensor solution of a corresponding lower scale boundary value problem, which represents the micro-scale heterogeneity. The boundary conditions of the lower scale problems are therefore dependent on the boundary conditions of the higher scale problem and are iteratively modified until the governing equations of the higher scale problem are satisfied, with each modification driving a new lower scale simulation. The order of the data-passing scheme (first or second) determines the degree to which nonuniformity in the displacement and fluid pressure fields can be communicated. The results for this study indicate that there may be a need to represent these nonuniformities due to their impact on the quantification of chondrocyte mechanics. Therefore, future implementation of computational homogenization for multiscale biphasic cartilage problems may want to consider employing $2^{\text {nd }}$ order Taylor Series accuracy.

Also regarding bi-directional multiscale analysis, in particular computational homogenization theory, the size of the cellular-scale model needs to be verified to ensure that it is a representative volume element (RVE) (Gitman et al., 2007) for transitional zone cartilage. This is of particular importance, as the accuracy of data passing schemes may be influenced by RVE size; larger RVEs may require higher order data passing (Kouznetsova et al., 2004). Presently, a study to characterize this for any cartilage zone at any level of constitutive complexity is generally lacking in the discipline. For multiscale biphasic analysis, there are additional fundamental barriers to overcome the determination of an RVE size, due to the dependence of mechanical time history on construct volume. All these issues need to be addressed before extending the analysis of chondrocyte mechanics into a bidirectional computational homogenization framework.

\section{Conclusion}

This study provided a general purpose framework for a post-processing based multiscale and multiphysics analysis to appropriately quantify cellular deformations from tissue level mechanical information. The sensitivity of cell level metrics to approximations in data 
passing were established along with estimates of accuracy. The study, although entirely theoretical in design, addressed the fundamental need to verify this post-processing based multiscale simulation approach. In the future, this evaluation pipeline can be expanded to incorporate other geometric and material nonlinearities, as required by the specifics of the tissue of interest. Validation approaches, $e . g$., comparison of predictions of cell mechanics to recently emerging experimental data (Abusara et al., 2011) will certainly establish increased confidence in predictions of multiscale analysis as well.

There are several advantages of the outlined post-processing approach, in addition to its cost-effectiveness. First, tissue level mechanical field variables (to be passed to cell level models) are only needed at a single point. Second, by increasing the order of information passed at a given point, i.e., with deformation and fluid pressure gradients and Laplacians, potential non-uniformity of boundary conditions of the cell level models can be represented. Third, the calculations of cell level metrics were automated and incorporated a wide range of variables (not just change in cell aspect ratio), which may provide insight into cell mechanobiology and damage. Given these advantages, previous multiscale analysis of cell deformations for large regions of cartilage (Sibole and Erdemir, 2012) can be extended to accommodate for the multiphysics nature of this tissue's behavior and to understand the functional mechanical environment of chondrocytes. If tissue level information can be obtained experimentally, e.g., as shown for meniscus (Upton et al., 2008) and for constructs (Bell et al., 2012), the post-processing approach may serve as a general purpose tool to associate tissue scale experimental measurements to cell mechanics. In return, understanding multiscale interactions between functional tissue behavior, cell mechanobiology and tissue and cell viability will be possible (Shoham and Gefen, 2012).

\section{Dissemination}

A download package incorporating the embedded and autonomous models, postprocessing scripts, and simulation results is freely accessible in the 'Downloads' section of the project web site https://simtk.org/home/j2c.

\section{Supplementary Material}

Refer to Web version on PubMed Central for supplementary material.

\section{Acknowledgments}

This study was funded by the National Institute of Biomedical Imaging and Bioengineering, National Institutes of Health (R01EB009643: Erdemir, Principal Investigator; R01EB015133: Weiss, Principal Investigator) and by the National Institute of General Medical Sciences, National Institutes of Health (R01GM083925: Weiss \& Ateshian, Co-Principal Investigators). The authors also acknowledge computing resources provided by the Ohio Supercomputer Center.

\section{References}

Abusara Z, Seerattan R, Leumann A, Thompson R, Herzog W. A novel method for determining articular cartilage chondrocyte mechanics in vivo. J Biomech. 2011; 44:930-934. [PubMed: 21145552] 
Alexopoulos LG, Setton LA, Guilak F. The biomechanical role of the chondrocyte pericellular matrix in articular cartilage. Acta Biomater. 2005; 1:317-325. [PubMed: 16701810]

Altman DG, Bland JM. Measurement in Medicine: The Analysis of Method Comparison Studies. The Statistician. 1983; 32:307.

Ateshian GA, Ellis BJ, Weiss JA. Equivalence between short-time biphasic and incompressible elastic material responses. J Biomech Eng. 2007; 129:405-412. [PubMed: 17536908]

Ateshian GA, Rajan V, Chahine NO, Canal CE, Hung CT. Modeling the matrix of articular cartilage using a continuous fiber angular distribution predicts many observed phenomena. J Biomech Eng. 2009; 131:061003. [PubMed: 19449957]

Bell BJ, Nauman E, Voytik-Harbin SL. Multiscale strain analysis of tissue equivalents using a customdesigned biaxial testing device. Biophys. J. 2012; 102:1303-1312. [PubMed: 22455913]

Cao L, Guilak F, Setton LA. Three-dimensional finite element modeling of pericellular matrix and cell mechanics in the nucleus pulposus of the intervertebral disk based on in situ morphology. Biomech Model Mechanobiol. 2011; 10:1-10. [PubMed: 20376522]

Chen J, Irianto J, Inamdar S, Pravincumar P, Lee DA, Bader DL, Knight MM. Cell mechanics, structure, and function are regulated by the stiffness of the three-dimensional microenvironment. Biophys. J. 2012; 103:1188-1197. [PubMed: 22995491]

Fisher RA. On the "probable error" of a coefficient of correlation deduced from a small sample. Metron. 1921; 1:1-32.

Geers MGD, Kouznetsova VG, Brekelmans WAM. Multi-scale computational homogenization: Trends and challenges. Journal of Computational and Applied Mathematics. 2010; 234:21752182.

Gitman IM, Askes H, Sluys LJ. Representative volume: Existence and size determination. Engineering Fracture Mechanics. 2007; 74:2518-2534.

Goldsmith AA, Hayes A, Clift SE. Application of finite elements to the stress analysis of articular cartilage. Med Eng Phys. 1996; 18:89-98. [PubMed: 8673324]

Grodzinsky AJ, Levenston ME, Jin M, Frank EH. Cartilage tissue remodeling in response to mechanical forces. Annu Rev Biomed Eng. 2000; 2:691-713. [PubMed: 11701528]

Guilak F, Mow VC. The mechanical environment of the chondrocyte: a biphasic finite element model of cell-matrix interactions in articular cartilage. J Biomech. 2000; 33:1663-1673. [PubMed: 11006391]

Halloran JP, Sibole S, Van Donkelaar CC, Van Turnhout MC, Oomens CWJ, Weiss JA, Guilak F, Erdemir A. Multiscale mechanics of articular cartilage: potentials and challenges of coupling musculoskeletal, joint, and microscale computational models. Ann Biomed Eng. 2012; 40:24562474. [PubMed: 22648577]

Holmes MH, Mow VC. The nonlinear characteristics of soft gels and hydrated connective tissues in ultrafiltration. J Biomech. 1990; 23:1145-1156. [PubMed: 2277049]

Hunziker EB, Quinn TM, Häuselmann H-J. Quantitative structural organization of normal adult human articular cartilage. Osteoarthr. Cartil. 2002; 10:564-572. [PubMed: 12127837]

Ihaka R, Gentleman R. R: A Language for Data Analysis and Graphics. Journal of Computational and Graphical Statistics. 1996; 5:299-314.

Julkunen P, Wilson W, Jurvelin JS, Korhonen RK. Composition of the pericellular matrix modulates the deformation behaviour of chondrocytes in articular cartilage under static loading. Med Biol Eng Comput. 2009; 47:1281-1290. [PubMed: 19898885]

Kouznetsova VG, Geers MGD, Brekelmans WAM. Multi-scale second-order computational homogenization of multi-phase materials: a nested finite element solution strategy. Computer Methods in Applied Mechanics and Engineering. 2004; 193:5525-5550.

Lai WM, Hou JS, Mow VC. A triphasic theory for the swelling and deformation behaviors of articular cartilage. J Biomech Eng. 1991; 113:245-258. [PubMed: 1921350]

Maas SA, Ellis BJ, Ateshian GA, Weiss JA. FEBio: finite elements for biomechanics. J Biomech Eng. 2012; 134:011005. [PubMed: 22482660]

Mononen ME, Mikkola MT, Julkunen P, Ojala R, Nieminen MT, Jurvelin JS, Korhonen RK. Effect of superficial collagen patterns and fibrillation of femoral articular cartilage on knee joint mechanicsa 3D finite element analysis. J Biomech. 2012; 45:579-587. [PubMed: 22137088] 
Moo EK, Herzog W, Han SK, Abu Osman NA, Pingguan-Murphy B, Federico S. Mechanical behaviour of in-situ chondrocytes subjected to different loading rates: a finite element study. Biomech Model Mechanobiol. 2012; 11:983-993. [PubMed: 22234779]

Mow VC, Ateshian GA, Spilker RL. Biomechanics of diarthrodial joints: a review of twenty years of progress. J Biomech Eng. 1993; 115:460-467. [PubMed: 8302026]

Peeters EAG, Bouten CVC, Oomens CWJ, Bader DL, Snoeckx LHEH, Baaijens FPT. Anisotropic, three-dimensional deformation of single attached cells under compression. Ann Biomed Eng. 2004; 32:1443-1452. [PubMed: 15535061]

Schwartz MA. Integrins and extracellular matrix in mechanotransduction. Cold Spring Harb Perspect Biol. 2010; 2:a005066. [PubMed: 21084386]

Shoham N, Gefen A. Deformations, mechanical strains and stresses across the different hierarchical scales in weight-bearing soft tissues. J Tissue Viability. 2012; 21:39-46. [PubMed: 22520396]

Sibole, S.; Bennetts, C.; Erdemir, A. A comparison of hexahedral and tetrahedral finite elements for biphasic analysis of cartilage micromechanics.. Presented at the ASME Summer Bioengineering Conference; Fajardo, Puerto Rico. 2012.

Sibole SC, Erdemir A. Chondrocyte deformations as a function of tibiofemoral joint loading predicted by a generalized high-throughput pipeline of multi-scale simulations. PLoS ONE. 2012; 7:e37538. [PubMed: 22649535]

Soltz MA, Ateshian GA. Experimental verification and theoretical prediction of cartilage interstitial fluid pressurization at an impermeable contact interface in confined compression. J Biomech. 1998; 31:927-934. [PubMed: 9840758]

Soulhat J, Buschmann MD, Shirazi-Adl A. A fibril-network-reinforced biphasic model of cartilage in unconfined compression. J Biomech Eng. 1999; 121:340-347. [PubMed: 10396701]

Spearman C. The proof and measurement of association between two things. Int J Epidemiol. 2010; 39:1137-1150. [PubMed: 21051364]

Upton ML, Gilchrist CL, Guilak F, Setton LA. Transfer of macroscale tissue strain to microscale cell regions in the deformed meniscus. Biophys. J. 2008; 95:2116-2124. [PubMed: 18487290]

Upton ML, Guilak F, Laursen TA, Setton LA. Finite element modeling predictions of region-specific cell-matrix mechanics in the meniscus. Biomech Model Mechanobiol. 2006; 5:140-149. [PubMed: 16520958]

Urban JP. Present perspectives on cartilage and chondrocyte mechanobiology. Biorheology. 2000; 37:185-190. [PubMed: 10912191]

Wang CC-B, Guo XE, Sun D, Mow VC, Ateshian GA, Hung CT. The functional environment of chondrocytes within cartilage subjected to compressive loading: a theoretical and experimental approach. Biorheology. 2002; 39:11-25. [PubMed: 12082263]

Yan KC, Nair K, Sun W. Three dimensional multi-scale modelling and analysis of cell damage in cellencapsulated alginate constructs. J Biomech. 2010; 43:1031-1038. [PubMed: 20096842] 


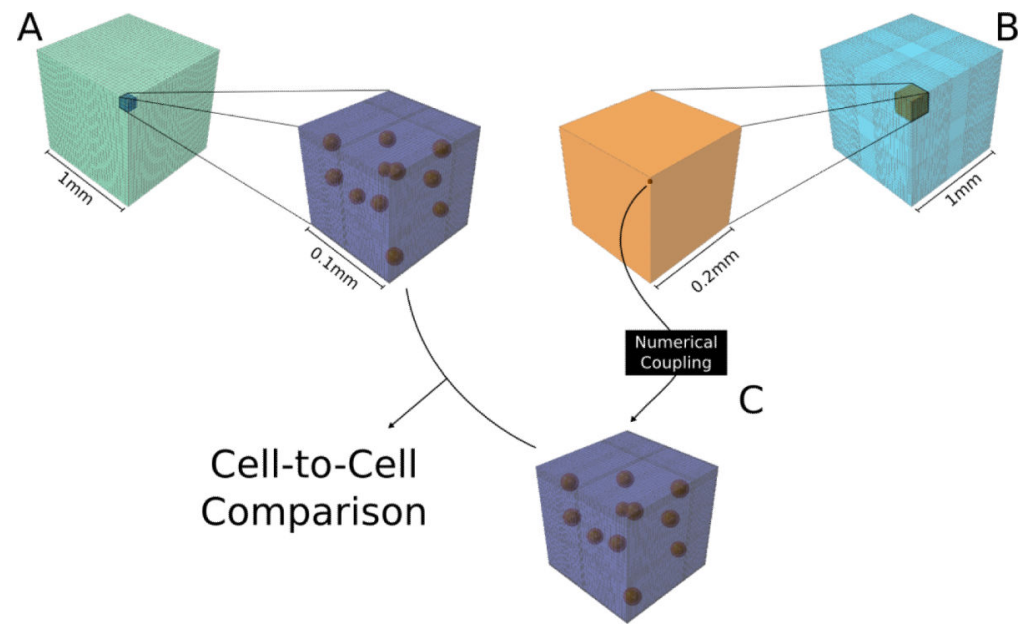

Figure 1.

An embedded (control) model including a region with representation of chondrocyte distribution and properties (A) is shown along with autonomous models: tissue level homogeneous model of cartilage (B) and a cell-scale model, which is identical to the region of the embedded model that has chondrocyte representation $(C)$. Simulations using the embedded model directly provide information on cell mechanics. When using autonomous models, first the tissue level model is solved. Following extraction of deformation and fluid pressure metrics (using $1^{\text {st }}$ or $2^{\text {nd }}$ order data passing) (C), the cell level model is solved to obtain chondrocyte mechanics. Performance of $1^{\text {st }}$ and $2^{\text {nd }}$ order coupling can be evaluated by comparing cell level metrics of autonomous simulations against those of the embedded model. 

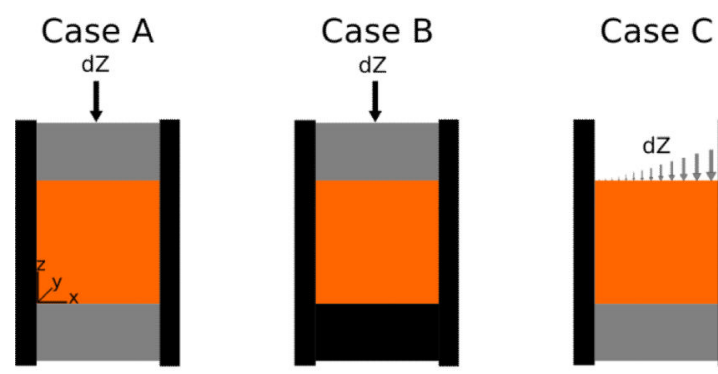

\section{Case D}

Load Curve

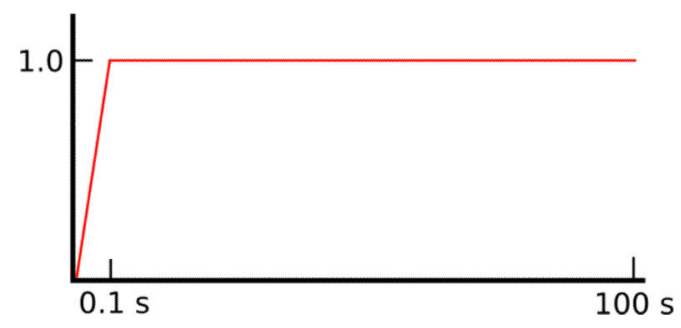

Tissue

Perfectly

Permeable Rigid

Impermeable

Rigid

Figure 2.

Simulation scenarios at increasing level of complexity in loading and boundary conditions are used to evaluate the performance of $1^{\text {st }}$ and $2^{\text {nd }}$ order data passing to acquire information on chondrocyte mechanics. Case A. Uniform displacement with symmetric permeability conditions. Case B. Uniform displacement with asymmetric permeability conditions. Case C. Non-uniform displacement with symmetric permeability conditions. Case D. Nonuniform displacement with asymmetric permeability conditions. Also illustrated is the time history of the of compressive displacement (dZ). For Cases A and B this displacement time history curve is scaled by -0.1 . For Cases $\mathrm{C} \& \mathrm{D}$, it is scaled by the solution of a nonuniform displacement function imitating a curved indenter. 


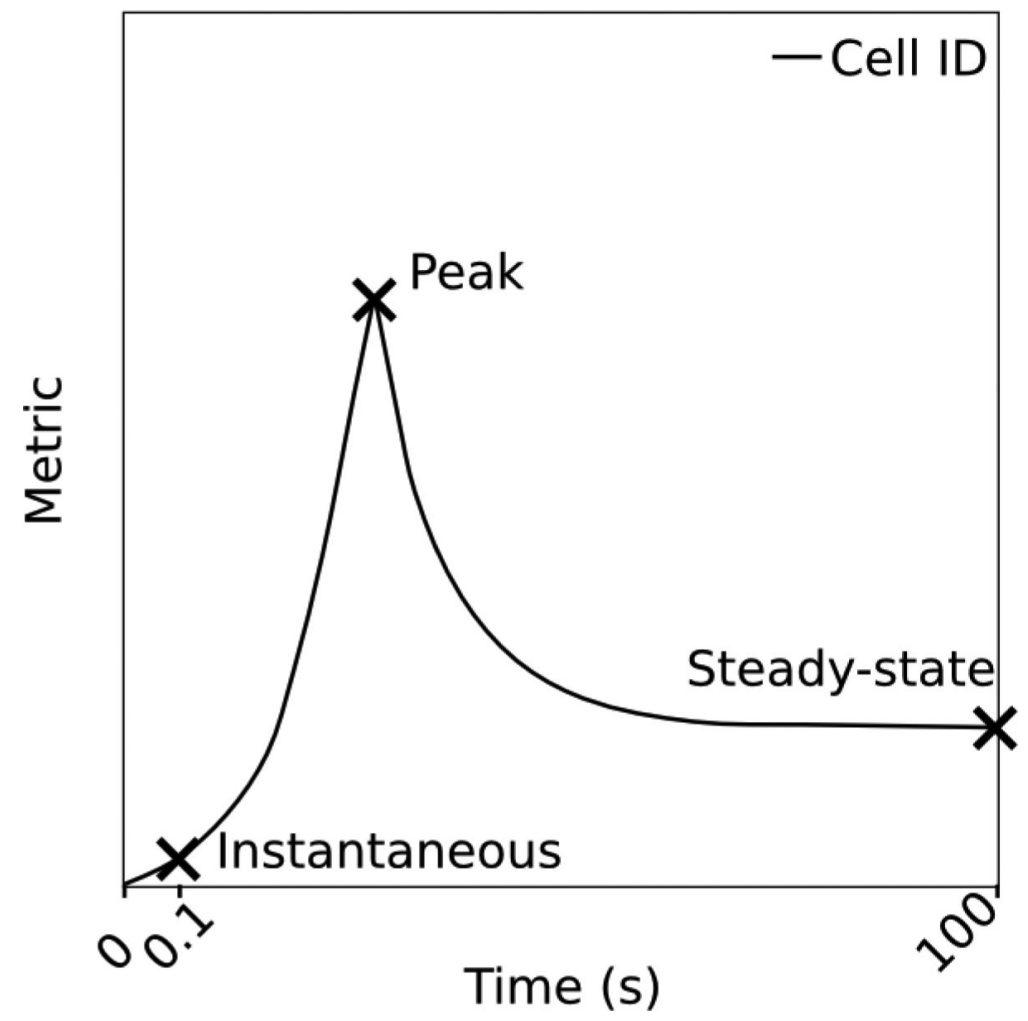

Figure 3.

For each cell, metrics of mechanics were extracted for two time points representative of "instantaneous" response (at peak loading of tissue level model) and "steady-state" response. The maximum of each mechanical metric, denoted as "peak", was also extracted irrespective of its occurrence throughout the course of simulation. 


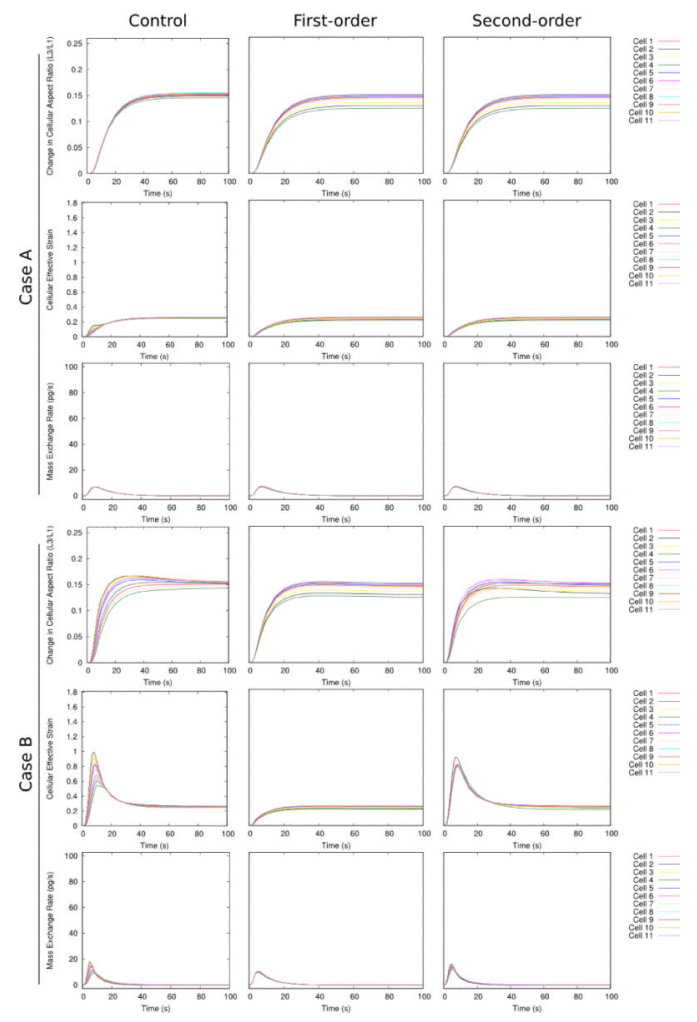

Figure 4.

The time histories of exemplar mechanical metrics for each chondrocyte during uniform elastic loading with symmetric and asymmetric permeability conditions (Cases A \& B) are shown. Embedded model results are shown along with autonomous simulations with $1^{\text {st }}$ and $2^{\text {nd }}$ order data passing. 


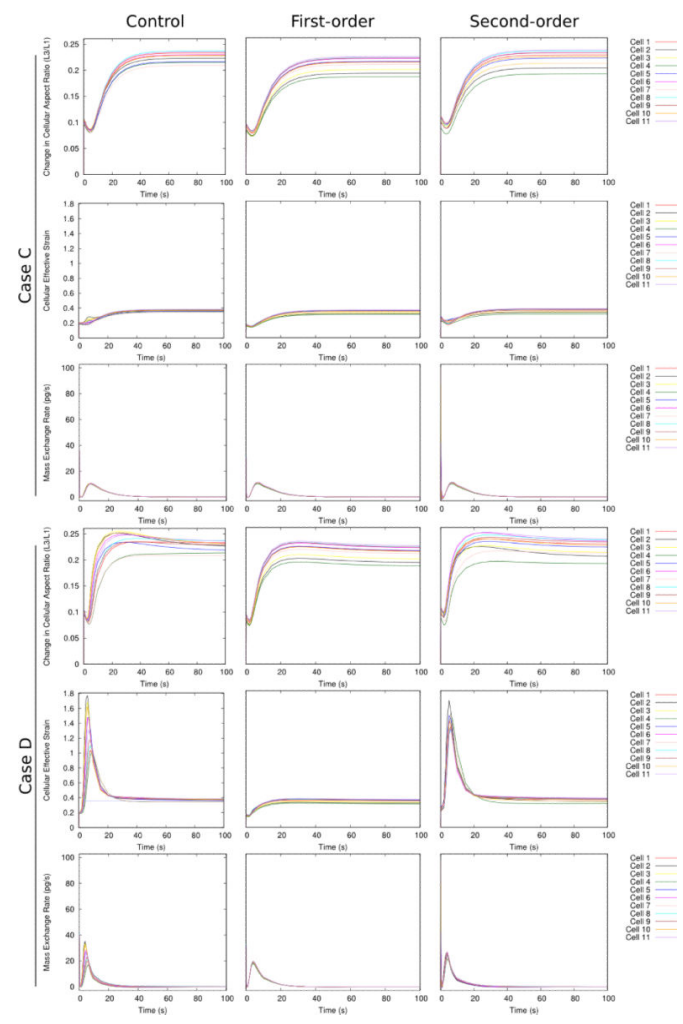

Figure 5.

The time histories of exemplar mechanical metrics for each chondrocyte during non-uniform elastic loading with symmetric and asymmetric permeability conditions (Cases C \& D) are shown. Embedded model results are shown along with autonomous simulations with $1^{\text {st }}$ and $2^{\text {nd }}$ order data passing. 
Change in Aspect Ratio

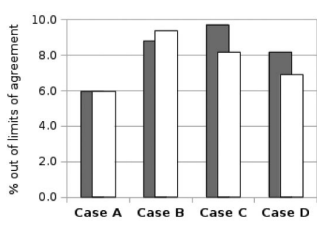

Effective Stress

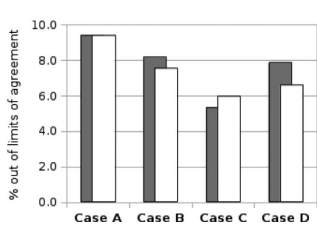

Volumetric Strain

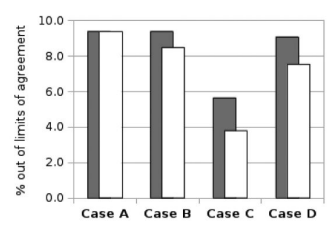

Maximum Shear Strain

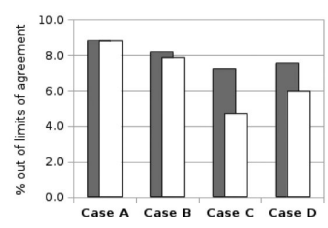

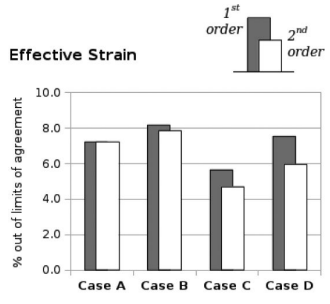

Mass Exchange Rate

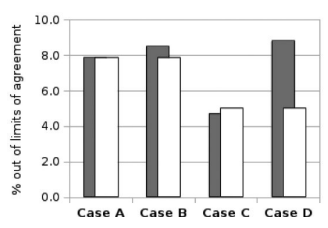

Figure 6.

The number of values lying outside the $95 \%$ Bland and Altman limits of agreement for each chondrocyte metric, when compared to control over the full simulation time, were normalized by the number of values in the set, $n=319$. 

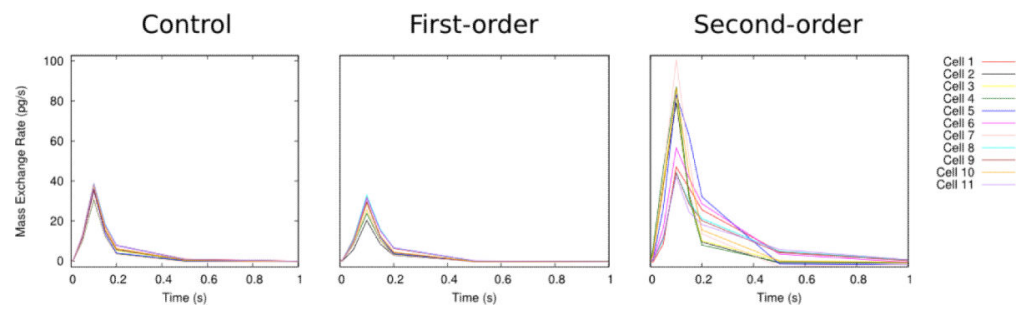

Figure 7.

The time histories of mass exchange rate during the first second of simulation Case $\mathrm{C}$ illustrate the potential over prediction of the instantaneous value of this metric when using $2^{\text {nd }}$ order biphasic simulations. Nonetheless, when the remainder of the time history for this variable and other solid mechanics metrics are also considered (Figures $4 \& 5$ ), $2^{\text {nd }}$ order data passing scheme for post-processing appears to be advantageous. 


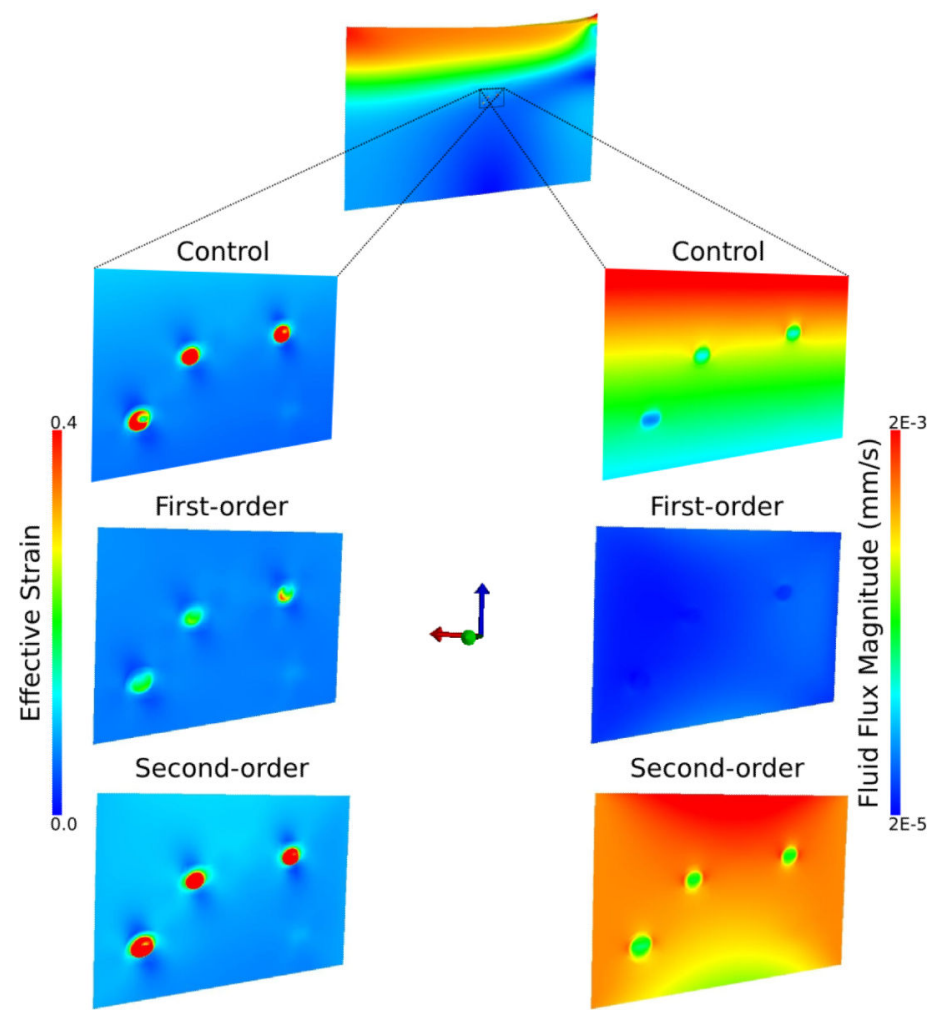

Figure 8.

A snapshot of the biphasic response of the embedded model cellular region and that obtained from autonomous simulations ( $1^{\text {st }}$ and $2^{\text {nd }}$ order) using the cell-scale model. Crosssectional distributions of effective strain are shown along with fluid flux for simulation Case $\mathrm{D}$ at $t=5 \mathrm{~s}$. 


\section{Table 1}

The material properties used in embedded and autonomous models for chondrocyte, pericellular matrix (PCM) and extracellular matrix (ECM) were obtained from (Guilak and Mow, 2000), which were originally derived from experimental studies. Note that tissue level cartilage representation is the same as cell level representation of the extracellular matrix.

\begin{tabular}{|c|c|c|c|}
\hline & Young's Modulus (kPa) & Poisson 's Ratio & Permeability $\left(\mathbf{m m}^{\mathbf{4} / \mathbf{N}-\mathbf{s})}\right.$ \\
\hline $\boldsymbol{E C M}$ & 1000 & 0.125 & 0.002 \\
\hline $\boldsymbol{P C M}$ & 43 & 0.125 & 0.002 \\
\hline Chondrocyte & 1 & 0.4 & 0.001 \\
\hline
\end{tabular}




\section{Table 2}

The root-mean-square differences between autonomous model (with $1^{\text {st }}$ or $2^{\text {nd }}$ order data passing) and embedded (control) model predictions of chondrocyte mechanical variables are given (see Equation 20). Normalized root-mean-square differences are also shown in parentheses. For information on simulation scenarios, please refer to Figure 2.

\begin{tabular}{|l|l|l|c|c|c|c|c|}
\hline Case & Data Passing Order & $\begin{array}{c}\text { Change in Cell } \\
\text { Aspect Ratio }\end{array}$ & Volumetric Strain & Effective Strain & Effective Stress (kPa) & $\begin{array}{c}\text { Maximum shear strain } \\
\text { Mass Exchange } \\
\text { Rate (pg/s) }\end{array}$ \\
\hline \multirow{2}{*}{$\boldsymbol{A}$} & First & $7.46 \mathrm{E}-3(0.048)$ & $9.02 \mathrm{E}-3(0.038)$ & $1.68 \mathrm{E}-2(0.064)$ & $1.44 \mathrm{E}-5(0.059)$ & $9.27 \mathrm{E}-3(0.070)$ & $6.23 \mathrm{E}-13(0.091)$ \\
\cline { 2 - 8 } & Second & $7.46 \mathrm{E}-3(0.048)$ & $9.02 \mathrm{E}-3(0.038)$ & $1.68 \mathrm{E}-2(0.064)$ & $1.44 \mathrm{E}-5(0.059)$ & $9.27 \mathrm{E}-3(0.070)$ & $6.23 \mathrm{E}-13(0.091)$ \\
\hline \multirow{2}{*}{$\boldsymbol{B}$} & First & $1.33 \mathrm{E}-2(0.083)$ & $1.82 \mathrm{E}-2(0.070)$ & $2.42 \mathrm{E}-1(0.318)$ & $1.45 \mathrm{E}-4(0.303)$ & $1.29 \mathrm{E}-1(0.319)$ & $1.75 \mathrm{E}-12(0.129)$ \\
\cline { 2 - 8 } & Second & $1.19 \mathrm{E}-2(0.074)$ & $1.45 \mathrm{E}-2(0.056)$ & $9.76 \mathrm{E}-2(0.128)$ & $5.52 \mathrm{E}-5(0.115)$ & $5.13 \mathrm{E}-2(0.127)$ & $1.74 \mathrm{E}-12(0.128)$ \\
\hline \multirow{2}{*}{$\boldsymbol{F}$} & First & $1.22 \mathrm{E}-2(0.052)$ & $1.38 \mathrm{E}-2(0.040)$ & $3.04 \mathrm{E}-2(0.079)$ & $2.75 \mathrm{E}-5(0.066)$ & $1.69 \mathrm{E}-2(0.087)$ & $1.94 \mathrm{E}-12(0.051)$ \\
\cline { 2 - 8 } & Second & $1.10 \mathrm{E}-2(0.047)$ & $1.79 \mathrm{E}-2(0.053)$ & $2.46 \mathrm{E}-2(0.064)$ & $2.17 \mathrm{E}-5(0.052)$ & $1.30 \mathrm{E}-2(0.067)$ & $9.75 \mathrm{E}-12(0.255)$ \\
\hline \multirow{2}{*}{$\boldsymbol{D}$} & First & $2.07 \mathrm{E}-2(0.083)$ & $2.90 \mathrm{E}-2(0.078)$ & $4.09 \mathrm{E}-1(0.312)$ & $1.98 \mathrm{E}-4(0.281)$ & $2.14 \mathrm{E}-1(0.311)$ & $3.70 \mathrm{E}-12(0.091)$ \\
\cline { 2 - 8 } & Second & $1.83 \mathrm{E}-2(0.074)$ & $2.69 \mathrm{E}-2(0.073)$ & $1.75 \mathrm{E}-1(0.133)$ & $7.82 \mathrm{E}-5(0.111)$ & $8.97 \mathrm{E}-2(0.130)$ & $9.37 \mathrm{E}-12(0.231)$ \\
\hline
\end{tabular}




\section{Table 3}

The Spearman rank correlation coefficients for cell level mechanical metrics calculated between embedded model results (control) and autonomous simulation predictions ( $1^{\text {st }}$ and $2^{\text {nd }}$ order) are given. Time points represent peak response as well as at instantaneous and steady-state loading (see Figure 3). Correlation coefficients were omitted when the metric was near zero and bolded when there was a significant difference $(\mathrm{p}<0.05)$ between $1^{\text {st }}$ and $2^{\text {nd }}$ order. For information on simulation scenarios, please refer to Figure 2.

\begin{tabular}{|c|c|c|c|c|c|c|c|}
\hline \multicolumn{8}{|c|}{ Instantaneous } \\
\hline Case & Data Passing Order & $\begin{array}{r}\text { Change } \\
\text { in Cell } \\
\text { Aspect } \\
\text { Ratio }\end{array}$ & Volumetric Strain & Effective Strain & Effective Stress & Maximum Shear Strain & Mass Exchange Rate \\
\hline \multirow[t]{2}{*}{$\bar{A}$} & First & & & & & & \\
\hline & Second & & & & & & \\
\hline \multirow[t]{2}{*}{$\bar{B}$} & First & & & & & & 0.4727 \\
\hline & Second & & & & & & 0.6182 \\
\hline \multirow[t]{2}{*}{$C$} & First & 0.2727 & 0.7727 & 0.3273 & 0.3273 & 0.2727 & 0.6727 \\
\hline & Second & 0.4455 & -0.6000 & 0.6727 & 0.6636 & 0.7455 & -0.4636 \\
\hline \multirow[t]{2}{*}{$D$} & First & 0.4636 & 0.8545 & 0.5909 & 0.6182 & 0.5909 & 0.9273 \\
\hline & Second & 0.5545 & -0.6455 & 0.4909 & 0.4818 & 0.5818 & -0.7364 \\
\hline
\end{tabular}

\begin{tabular}{|c|c|c|c|c|c|c|c|}
\hline \multicolumn{8}{|c|}{ Peak } \\
\hline Case & Data Passing Order & $\begin{array}{r}\text { Change } \\
\text { in Cell } \\
\text { Aspect } \\
\text { Ratio }\end{array}$ & Volumetric Strain & Effective Strain & Effective Stress & Maximum Shear Strain & Mass Exchange Rate \\
\hline \multirow[t]{2}{*}{$\boldsymbol{A}$} & First & 0.8818 & 0.8636 & 0.9091 & 0.9091 & 0.9091 & 0.6182 \\
\hline & Second & 0.8818 & 0.8636 & 0.9091 & 0.9091 & 0.9091 & 0.6182 \\
\hline \multirow[t]{2}{*}{$B$} & First & 0.0182 & -0.0273 & 0.0000 & 0.0000 & 0.0000 & -0.0727 \\
\hline & Second & 0.2000 & 0.3909 & 0.6818 & 0.9364 & 0.7545 & 0.9364 \\
\hline \multirow[t]{2}{*}{ C } & First & 0.7000 & 0.6273 & 0.7182 & 0.7182 & 0.7273 & 0.6727 \\
\hline & Second & 0.7909 & 0.8364 & 0.7455 & 0.8273 & 0.8364 & -0.4636 \\
\hline \multirow[t]{2}{*}{$D$} & First & 0.0182 & -0.0273 & 0.0000 & 0.0000 & -0.0273 & 0.9273 \\
\hline & Second & 0.3545 & 0.3818 & 0.2000 & 0.7545 & 0.2000 & -0.7364 \\
\hline
\end{tabular}

\begin{tabular}{|c|c|c|c|c|c|c|c|}
\hline \multicolumn{8}{|c|}{ Steady State } \\
\hline Case & Data Passing Order & $\begin{array}{r}\text { Change } \\
\text { in Cell } \\
\text { Aspect } \\
\text { Ratio }\end{array}$ & Volumetric Strain & Effective Strain & Effective Stress & Maximum Shear Strain & Mass Exchange Rate \\
\hline \multirow[t]{2}{*}{$\boldsymbol{A}$} & First & 0.8818 & 0.8636 & 0.9091 & 0.9091 & 0.9091 & \\
\hline & Second & 0.8818 & 0.8636 & 0.9091 & 0.9091 & 0.9091 & \\
\hline \multirow[t]{2}{*}{$B$} & First & 0.6091 & 0.2182 & 0.8091 & 0.7182 & 0.7545 & \\
\hline & Second & 0.7091 & 0.4818 & 0.8545 & 0.7818 & 0.7818 & \\
\hline \multirow[t]{2}{*}{$C$} & First & 0.7000 & 0.6273 & 0.7182 & 0.7182 & 0.7273 & \\
\hline & Second & 0.7909 & 0.8364 & 0.7455 & 0.8273 & 0.8364 & \\
\hline
\end{tabular}


Steady State

\begin{tabular}{|c|c|c|c|c|c|c|c|}
\hline Case & Data Passing Order & $\begin{array}{r}\text { Change } \\
\text { in Cell } \\
\text { Aspect } \\
\text { Ratio }\end{array}$ & Volumetric Strain & Effective Strain & Effective Stress & Maximum Shear Strain & Mass Exchange Rate \\
\hline \multirow[t]{2}{*}{$\bar{D}$} & First & 0.6636 & 0.5545 & 0.7364 & 0.6455 & 0.7364 & \\
\hline & Second & 0.8273 & 0.7909 & 0.7364 & 0.7091 & 0.8273 & \\
\hline
\end{tabular}

